ISBN 978-981-14-1684-2

Proceedings of 2019 the 9th International Workshop on Computer Science and Engineering

(WCSE 2019 SUMMER)

Hong Kong, 15-17 June, 2019, pp. 891-896

doi: $10.18178 /$ wcse. 2019.06 .133

\title{
User Perspective on the Generation Gap in Using Internet of Things - IoTs: A Conceptual Framework
}

\author{
Wornchanok Chaiyasoonthorn, Kulapa Najantong and Singha Chaveesuk ${ }^{+}$ \\ Faculty of Administration and Management \\ King Mongkut's Institute of Technology Ladkrabang, Bangkok, Thailand
}

\begin{abstract}
Rapid changes in technology have caused many changes in business conduct. At present, several technological revolutions are happening such as smart-city, smart-health, smart-traffic, and smart-device. The operations of all of these smart things are based on wireless sensor network connecting them and other machines together. We called them collectively as Internet of Things or IoTs. IoTs technology disrupts businesses and has a huge impact on all generations of humans. New technologies bring with them some useful functionality. However, usually, there is a gap between the full capacity of new technology and its utilization by users. This study explores the conceptual framework of user perspective on generation gap in using IoTs technology, based on Expected Disconfirmation, Educational Technology Gap, and Trust theories. The authors hope that this framework will benefit other researchers in terms of potential factors influencing IoTs technology usage continuance intention in their further research on this issue.
\end{abstract}

Keywords: internet of things ; expected disconfirmation theory; educational technology gap theory; trust theory;

\section{Introduction}

In recent years, the emerging technologies pertaining to internet protocols and computing systems have made communication between various connected devices more efficient than ever before [1]. According to [1], around 25-50 billion devices are expected to be connected to the Internet by 2020. These connected devices and the connections between them via the internet are called Internet of Things - IoTs. Today, it is increasingly famous as rapid progress in the fields of ubiquitous computing, Wireless Sensor Network (WSN) and Machine-to-Machine (M2M) based communication [2]. The term Internet of Things (IoTs) was coined in 1999 by a British technology pioneer, Kevin Ashton, to describe a system in which objects in the physical world could be connected to the Internet via sensors. For instance, Radio-Frequency IDentification (RFID) tags on pieces of products can be used to count the number of available products and their inventory can be tracked automatically without the need for any human actions [3]. Today, Internet of Things is becoming a popular term for internet connectivity and computing capability extends to a variety of objects, devices, sensors, and day-to-day life items [3].

IoTs communication protocols can be divided into three major groups [1]:

1. Device to Device (D2D): the protocols for the next generation of cellular networks;

2. Device to Server (D2S): the protocols for cloud processing;

3. Server to Server (S2S): the protocols for communication between servers.

The key IoTs utility factors include the capability to dynamically adapt to various connected devices with self-adapting and self-configuring capability, allowing a large number of devices to work together. Its interoperable communication protocols enable efficient communication between devices and the infrastructure. Every IoTs device has a unique identity and unique identifiers such as an IP address or a URL. IoTs is integrated with information networks. It is capable of context-awareness that is based on the sensed

\footnotetext{
+ Corresponding author. Tel.: +663298459 fax: +663298461
}

E-mail address: singha@it.kmitl.ac.th. 
information and intelligent decision-making capability [4].

IoTs supports the following technologies: existing hardware platforms such as processor, clock Speed, bus width, system memory, communication supported, development environments, and programming languages; wireless communication standards such as protocols that define data exchange formats, data encoding, addressing schemes for devices and routing sources; cloud technology solutions such as real-time data capture, visualization, data analytics, decision making, and device management; and application-specific domains such as "application development, device management, and system management [4].

Today, IoTs can be found everywhere with devices, systems, and infrastructures such as RFID, NFC, wireless sensor network, environment monitoring infrastructure, infrastructure monitoring application, supply chain management, health care, hospital information system, smart society, smart city, smart home, smart health, cloud service and management, sensor discovery service, big data, data filtering, social computing, security issue, and virtual resources (a management concept that identifies different approaches in the performance evaluation of edge computing devices) $[1,4,5,6]$.

As mentioned above, digital technology is increasingly becoming incorporated in every product and process in everyday life [7]. Digital disruption is highly visible in the news and media industry. It may even topple large enterprises and significantly disrupt established industries [7]. The generation gap is one of the barriers between users and this disrupting technology. For instance, the gap between baby boomer and Gen Z may create different usage problems from one to the other $[8,9]$. Common problems in the scenario of the Internet of Things include connections between smart devices cannot be established in advance and some devices cannot be discovered [10]. Younger generations better use the latest technologies and can absorb their impact well [11]. Since IoTs is relatively new, a large number of surveys have identified a variety of security issues and challenges found in IoT systems [11].

The IoTs evolution has created its own security and privacy challenges. Most of these challenges come from the inherent vulnerabilities of IoT objects and the tight coupling of the physical world to the virtual world through intelligent objects [12]. Therefore, security and privacy challenges and solutions that lie ahead are discussed both in general and in the context of the system [13]. Privacy, trust, and sensitivity are of serious concerns among users of IoTs [14]. An example of good service applications that embodied 'trust', 'privacy', and 'sensitivity' is an assistive care service application reported in [15]. In contrast, lack of trust can be attributed to exclusion of users from service design choices-making their engagement with technology more difficult than they expected [15].

This study proposed a conceptual framework of user perspective on the generation gap in using IoTs. The conceptual framework explains the gap between generations in their satisfaction of IoTs usage and is based on the following ideas: Oliver's expected confirmation theory [16]; the educational technology gap model of Bohler et al. [17]; Ybarra and Park's findings of disconfirmation of person expectations of older and younger adults [18], and Expected disconfirmation theory (EDT) and a model of trust in technology of Lankton et al [19]. Next section reviews these ideas.

\section{Literature Review and Hypothesis Development}

\subsection{Expected Disconfirmation Theory}

Am Expectation Disconfirmation Theory (EDT) was proposed by Oliver R.L. in 1980 [16]. This theory deals with expectations and perceived performance of any product or service which can predict user satisfaction of them [16, 17]. This can be related to hypothesis 2 as technology trusting expectation positively influences technology trusting performance (see Fig.4). Positive or negative disconfirmation is directly related to better or worse performance, which can be seen in hypothesis 4 as technology trusting performance positively influences technology trusting disconfirmation/Gap (see Fig. 4). Both affect users' degree of satisfaction [16, 17]. A chart of Oliver's EDT is shown in Fig. 1. Technical papers that cited EDT are such as "Incorporating trust-in-technology into Expectation Disconfirmation Theory" [19] that explained that users' trust in a system might affect their satisfaction and continuance intention. Another example is "Intimacy, familiarity and continuance intention: An extended expectation-confirmation model in web-based services" [20] that investigated an expectation-confirmation model (ECM) and explains why and how 
consumers are motivated to continue to use web-based services and their satisfaction of them. These refer to hypothesis 5,6,7, and 10 as follows: 1) technology trusting disconfirmation and gap positively influences technology trusting intention; 2) technology trusting disconfirmation and gap positively influences technology Satisfaction 3) technology satisfaction positively influences technology trusting intention; 4) control variables perceived usefulness influences technology trusting intention; 5) control variables perceived ease of use influences technology trusting intention; and 6) technology trusting intention positively influences IoTs technology usage continuance intention (see Fig. 4).

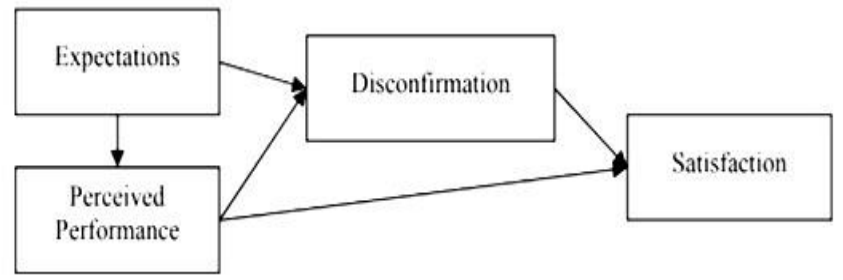

Fig. 1: Oliver's expected disconfirmation theory (EDT) cited by Bohler, et al., 2014.

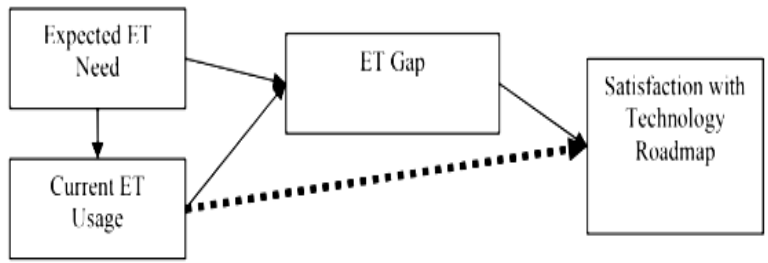

Fig. 2: The educational Technology Gap Model of Bohler, et al., 2009.

\subsection{Educational Technology Gap Theory}

Bohler, et al. proposed an educational technology gap theory (ET) in 2009. They modified EDT by introducing a generation gap variable into it as shown in Fig. 2 and technology trusting disconfirmation/gap variable shown in Fig. 4 [17]. ET adopted EDT to explain the dissonance between the current ET usage at that time and the having been expected ET usage. The explanation would lead to studies of factors affecting 'satisfaction with technology roadmap' [17]. Their ET contributed to the creation of the conceptual framework in this study. Another contribution was from the idea of Michele Jacobsen and François Desjardins (2007) that "The Middle English word gap descends from the Old Norse for chasm, both of which denote a marked interruption of continuity. Educational technology research is framed by questions and/or hypotheses and is often conducted through empirical, narrative and/or descriptive means to uncover or revise understanding by identifying persistent trends, offering authoritative explanations, creating models, outlining theories, designing applications and reflecting on values, beliefs and purpose" [21]. As an aside, educational technology consisted of multidisciplinary activities that were enjoying a period of growth at that time [22].

\subsection{Generation Gap}

Aggarwal (2017) mentioned that the generation gap came with some serious problems observed in youths and their elder generations because of various factors [23]. The statistical results that Aggarwal found showed that there were some issues between different generations due to many reasons such as lack of communication, different perspectives, cultural variables, and effect of new technology [20]. Generations were classified as follows: baby boomers (1943-1964), generation X or Gen-Xers (1965-1977), and generation Y (Millennials) (1978-1990) [24]. The authors of [24], L. Lebioda and I. S. Hahn, included another newer generation, generation $\mathrm{Z}$ or Gen-Z of people born after the nineties [24]. It has been generally argued that there were issues in the use of information related to generation gap, and generation gap strongly influenced the adoption of technology and communication technologies [25]. One of the studies by Ybarra and Park, "Disconfirmation of Person Expectations by Older and Younger Adults: Implications for Social Vigilance" [18], has helped the authors of this paper confirm the relationship between age and our 'technology trusting expectation' variable. In addition, one finding from one of Ybarra and Park's studies [18] 
that "Participants from both age groups tended to preferentially process and remember information that was inconsistent rather than consistent with positive expectancies. However, when the participants held negative expectancies about the target, they tended to preferentially remember information that was consistent rather than inconsistent with the expectancies. The findings suggest that both younger and older adults aim to test and disconfirm a belief that an unknown other has a positive character but appear to want to maintain and reinforce a belief that an unknown other has a negative character" also supports the same previous conclusion. Hypothesis 1 (age positively influences technology trusting expectation) has been developed (see Fig. 4).

\subsection{Trust Theory}

Trust is an essential component of human relationship and a fundamental building block of healthy societies. [26]. Hill and O'Connor stated that "Trust experts all seem to agree that trust is a state of mind that enables its possessor to be willing to make herself vulnerable to another-that is, to rely on another despite a positive risk that the other will act in a way that can harm the truster"; "Although law and social norms can work to encourage "trust that" trust, it is, at best, a substitute for "trust in" trust"; "trust and distrust are assumed to lie along a unidimensional continuum, where the degree of trust is represented by the amount that a Player 1 is willing to send to her Player 2"; and "However, the relationship between trust and distrust calls the validity of the unidimensional characterization into question" [26]. Trust involves positive expectations about things hoped for [26]. Distrust involves positive expectations about things feared [26]. This refers to hypothesis 3as technology trusting expectation positively influences technology trusting disconfirmation/gap (see Fig. 3). Lewicki et al. [27] explored the integration of trust and distrust in alternative social realities. This research explains that the first dimension is low trust and low distrust which includes casual acquaintances, limited interdependence, bounded, arms-length transactions, and professional courtesy. The second dimension is high trust and low distrust which includes high-value congruence, interdependence promoted, opportunities pursued, and new initiatives. The third dimension is low trust and high distrust which includes undesirable eventualities expected and feared, harmful motives assumed, interdependence managed, pre-emption; best offense is a good defense, and paranoia. The fourth dimension is high trust and high distrust which includes trust but verify, relationships highly segmented and bounded, opportunities pursued, and down-side risks/vulnerabilities continually monitored.

TechUK stated that the IoTs can provide more benefits to the society and economy, driving better health services, cleaner and safer societies, more efficient industries and stimulating new businesses. To achieve those benefits, IoTs will leverage unprecedented volumes of detailed, and often real-time, data [28]. In addition to building consumer trust with regard to data, industries must acknowledge and build trust through high standards of physical safety. Where everyday actions are enhanced with the use of IoTs, it is important that consumers feel safe and trust the digitally enabled actions [28]. Trust has been found to affect user adoption of many electronic services (e.g. mobile shopping, online social networks, and internet banking) [29]. The framework in Fig. 3 is from Lankton et al. [19] integrating expected disconfirmation theory (EDT) with trust in technology. Moreover, two control variables have also been found as a significant effect to technology trusting intention seen as usefulness and ease of use. These can relate to hypothesis 8 and 9 (see Fig. 4). It contributed substantially to the construction of the proposed framework in this study.

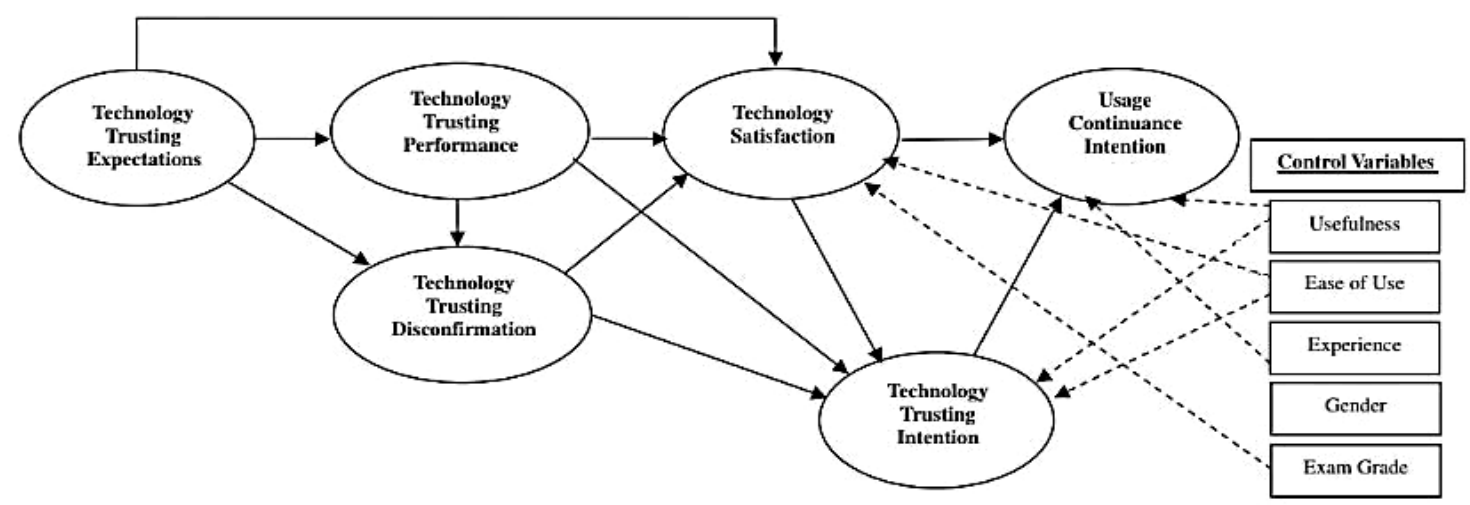

Fig. 3: The integrated expected disconfirmation theory (EDT) and trust in technology [19]. 


\section{Conceptual Framework and Hypothesis}

In order to build the conceptual framework, this research employed three main theories: EDT, ET, and Trust theories. The following ten hypotheses were constructed shown in Fig. 4. All hypotheses generated above have been discussed in literature review part.

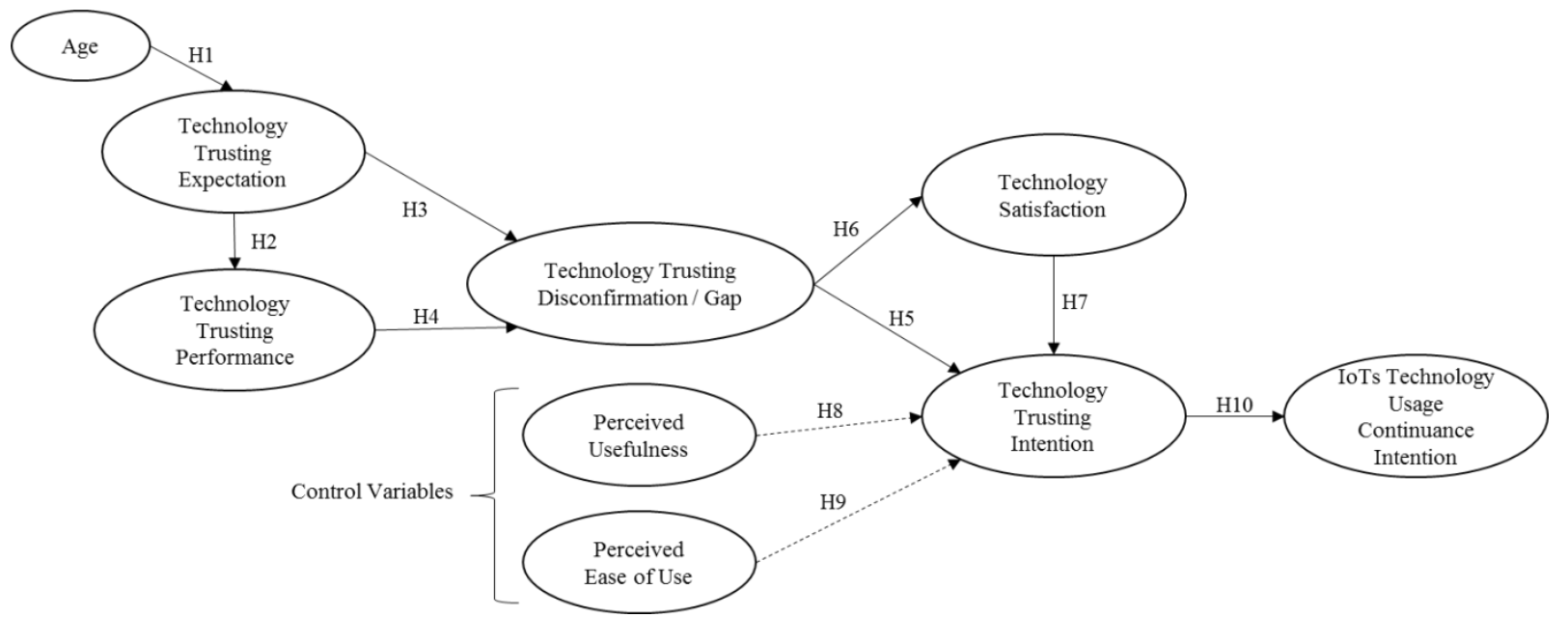

Fig. 4: The conceptual Framework formulated by EDT, ET, and trust in technology.

\section{Contributions and Further Study}

This research aimed to propose a conceptual framework that can explain user perspective on the generation gap in using Internet of Things (IoTs) in order to find potential factors influencing IoTs technology usage continuance intention. This theoretical framework is based on EDT, ET, and Trust theories, embodying massive prior knowledge. A suggested further study is collection of questionnaire data from people of various age groups representing various generations and analysis of the data by using a structural equation model (SEM).

\section{References}

[1] M. S. Mahdavinejad, M. Rezvan, M. Barekatain, P. Adibi, P. Barnaghi, and A. P. Sheth. Machine learning for Internet of Things data analysis: a survey. Digital Communications and Networks. 2018, vol. 4, pp. 161 - 175.

[2] M. Bilal. A Review of Internet of Things Architecture, Technologies and Analysis Smartphone-based Attacks Against 3D printers. Networking and Internet Architecture. 2017, pp. 1 - 21.

[3] K. Rose, S. Eldridge, and L. Chapin. The Internet of Things : An Overview Understanding the Issues and Challenges of a More Connected World. Internet Society. 2015, pp. 53.

[4] P. P. Ray. A survey on Internet of Things architectures. Journal of King Saud University - Computer and Information Sciences. 2016, vol. 30, pp. $291-319$.

[5] S. Mukherjee and G. P. Biswas. Networking for IoT and applications using existing communication technology. Egyptian Informatics Journal. 2018, vol. 19, pp. 107 - 127.

[6] M. Samaniego and R. Deters. Management and Internet of Things . Procedia Computer Science. 2016, vol. 94, pp. $137-143$.

[7] T. Saarikko, U. H. Westergren, and T. Blomquist. The Internet of Things : Are you ready for what's coming?. Business Horizons. 2017, vol. 60, pp. 667 - 676.

[8] S. Wiley. (2019). Understanding Today's Workforce: Generational Differences and the Technologies They Use. Available: https://www.firmofthefuture.com/content/understanding-todays-workforce-generational-differencesand-the-technologies-they-use/.

[9] P. Rue. Make Way, Millennials, Here Comes Gen Z. Sage Journals. 2018, vol. 23, pp. 5 - 12.

[10] S. Efremov, N. Pilipenko, and L. Voskov. An Integrated Approach to Common Problems in the Internet of Things. Procedia Engineering. 2015, vol. 100, pp. 1215 - 1223.

[11] O. Mavropoulos, H. Mouratidis, A. Fish, E. Panaousis, and C. Kalloniatis. A Conceptual Model to Support Security 
Analysis in the Internet of Things . Computer Science and Information Systems. 2017, vol.14, pp.557- 578.

[12] A. R. Sfar, E. Natalizio, Y. Challal, and Z. Chtourou. A roadmap for security challenges in the Internet of Things . Digital Communications and Networks. 2018, vol. 4, pp. 118 - 137.

[13] C. Maple. Security and privacy in the Internet of Things . Journal of Cyber Policy. 2017, vol. 2, pp. 155 - 184. [14] J. Daubert, A. Wiesmaier, and P. Kikiras. A View on Privacy \& Trust in IoT. 2015, presented at the 2015 IEEE

[14] International Conference on Communication Workshop (ICCW), London, UK.

[15] S. Bhattacharya, D. Wainwright, and J. Whalley. Internet of Things (IoT) enabled assistive care services: Designing for value and trust. International Workshop on Universal Design for IoT Smart Health (UDISH 2017). 2017, vol. 113, pp. 659-664.

[16] R. L. Oliver. A Cognitive Movel of the Antecedents and Consequences of Satisfaction Decisions. Journal of Marketing Research. 1980, vol. 17, pp. 460-469.

[17] J. A. Bohler, F. K. Weigel, and D. J. Hall. Educational Technology Gap Theory. 15th Americas Conference on Information Systems. 2009, San Francisco, California, pp. 1-10.

[18] O. Ybarra and D. C. Park. Disconfirmation of Person Expectations by Older and Younger Adults: Implications for Social Vigilance. Journal of Gerontology: Psychological Sciences. 2002, vol. 57B, pp. 435-443.

[19] N. Lankton, D. H. McKnight, and J. B. Thatcher. Incorporating trust-in-technology into Expectation Disconfirmation Theory. Journal of Strategic Information Systems. 2014, vol. 23, pp. 128-145.

[20] Y. Lee and O. Kwon. Intimacy, familiarity and continuance intention: An extended expectation-confirmation model in web-based services. Electronic Commerce Research and Applications. 2011, vol. 10, pp. 342-357.

[21] M. Jacobsen and F. Desjardins. Editorial: Filling the Gaps in Educational Technology Research. Canadian Journal of Learning and Technology. 2007, vol. 33.

[22] K. Issroff and E. Scanlon. Educational Technology: The Influence of Theory. Journal of Interactive Media in Education. 2002, vol. 6, pp. 1-13.

[23] M. Aggarwal, M. S. Rawat, S. Singh, S. Srivastava, and P. Gauba. Generation Gap: An Emerging Issue of Society. International Journal of Engineering Technology Science and Research. 2017, vol. 4, pp. 973-983.

[24] L. Lebioda and I. S. Hahn. Generation Gap: Does The Use of Mobile Technology Affect the Perceived Work Performance?. XL Encontro do ANPAD. 2016, pp. 1-13.

[25] A. Bailey and O. Ngwenyama. Bridging the Generation Gap in ICT Use: Interrogating Identity, Technology and Interactions in Community Telecenters. Information Technology for Development. 2010, vol. 16, pp. 62-82.

[26] C. Hill and E. O. H. O'Connor. A Cognitive Theory of Trust. Washington University Law Review. 2006, vol. 84, pp. 1717-1796.

[27] R. Lewicki and D. J. McAllister. Trust and Distrust: New Relationships and Realities. Academy of Management Review. 1998, vol. 23, pp. 438-458.

[28] [techUK. (2018), Trust in an Internet of Things World. Available: www.techuk.org.

[29] S. Chaveesuk, P. Wutthirong, and W. Chaiyasoonthorn. The Model of Mobile Payment System Acceptance on Social Networks in Thailand: A Conceptual Framework. presented at the the 2018 10th International Conference on Information Management and Engineering. 2018, Salford, United Kingdom, pp. 35-39. 